\title{
Adaptación de la industria del libro en España al cambio tecnológico Pasado, presente y futuro de la digitalización
}

\author{
Marta Magadán Díaz \\ Universidad Internacional de La Rioja, España | marta.magadan@unir.net / http://orcid.org/oooo-ooo3-3178-321
}

\author{
Jesús I. Rivas García \\ Universidad Internacional de La Rioja, España l jesus.rivas@unir.net / http://orcid.org/oooo-0oo3-0576-5961
}

\begin{abstract}
Resumen
El objetivo de este trabajo es analizar cómo afecta el cambio tecnológico a la industria editorial española y como esta se va adaptando al nuevo entorno digital. En el trabajo se ofrece una visión general de cuál es la situación del sector editorial en España a través de la recopilación de datos procedentes, principalmente, de fuentes de información secundaria, tanto pública como privada, desde el año 1996 hasta el 2017 , lo que ha supuesto revisar una gran cantidad de documentos de diferentes procedencias y agrupar los datos obtenidos, generando una serie temporal que permite vislumbrar cuál es el estado y la evolución de la digitalización en la industria editorial española en estos casi veinte años. En este documento se hace referencia a los nuevos modelos de negocio que surgen como consecuencia de la digitalización, para finalmente cerrar con unas conclusiones y limitaciones del trabajo. Este trabajo no deja sino de ser una muestra del pasado, presente y futuro de la industria del libro en España, pero supone el primer paso para continuar investigando otros aspectos de una manera más profunda.
\end{abstract}

\section{Adaptation of the book industry in Spain to technological change. Past, present and future of digitization}

\footnotetext{
Abstract

The main goal of this paper is to analyze how technological change affects the Spanish publishing industry and how it is adapting to the new digital environment. This work provides an averview of the situation of the Spanish publishing sector through the collection of data, mainly from secondary sources of information, both public and private, from 1996 to 2017, that involved the review of a large number of documents from different sources, the analysis of data obtained, and the generation of a time series allowing us to glimpse the state and evolution of digitalization in the Spanish publishing industry throughout these last twenty years. The paper refers to

\section{Palabras clave}

Digitalización Industria editorial Innovación Modelo de negocio Impresión bajo demanda Contenido digital e-Publicación Libro electrónico España

\section{Keywords}

Digitization Publishing industry Innovation Print on demand Business model Digital content ePublishing e-book Spain 
the new business models emerging as a result of digitization to, finally, close with some conclusions and limitations of the research. This study is not trying to be just a journey through the past, present and future of the spanish book industry in Spain, but it pretends to be the first step to continue investigating these and other aspects in a deeper way.

Artículo recibido: 19-06-2018. Aceptado: 06-03-2019

\section{Introducción}

La industria editorial, al igual que otras industrias creativas -como la musical (Hracs, 2012), la cinematográfica (Currah, 2006) y la publicitaria (Evans, 2008; Bugge 2011), está experimentando un proceso de cambio tecnológico que la enfrenta a nuevos desafíos (Donoughue, 2010). De hecho, el mundo editorial está sufriendo una profunda transformación a raíz de la transición digital, tanto en el plano del producto que la misma ofrece como en los procesos de producción y de distribución al mercado, la cual se extiende a lo largo de toda la cadena de valor del sector editorial (Bockstedt et al., 2006; Tian y Martin, 2010; Lichtenberg, 2011; Carolan y Evain, 2013; Galuszka, 2015). Concretamente, las empresas que en él operan han tenido que hacer frente a los nuevos retos planteados por las innovaciones y los cambios tecnológicos asociados a Internet, el ebook o la impresión bajo demanda, entre otros (Tian et al., 2008; Ho et al., 2011; Benghozi y Salvador, 2016).

Hoy en día, gracias a las tecnologías y a la posibilidad de subcontratar servicios y procesos industriales, tanto la opción de editar como de publicar un libro se hace cada vez más factible. Traducido en términos económicos, las barreras de entrada al sector son menores, lo que promueve una mayor diversificación de la oferta con la creación de nuevas empresas editoriales y facilitando la autopublicación. En estos momentos, se está dando la siguiente paradoja: por una parte, se conforman grandes estructuras editoriales, a través de fusiones y adquisiciones y, por otro lado, surgen iniciativas de pequeño tamaño, dando lugar a una atomización de la estructura del sector editorial español.

En España existen más de 3.000 empresas editoriales de capital privado (cuyo domicilio social y fiscal se encuentra radicado en el país), aunque la gran mayoría publican menos de 10 títulos anuales y su estructura se encuentra configurada mayoritariamente por pequeñas y medianas empresas, donde más de una cuarta parte de las cuales pertenece a algún grupo empresarial.

A efectos de este trabajo, se considerarán como editoriales españolas aquellas que cumplan simultáneamente con los siguientes requisitos: a) poseer personalidad jurídica propia constituida registralmente en España; b) poseer domicilio social y fiscal en España, por lo que, atendiendo a estos criterios, por ejemplo, Penguin Random House, se consideraría empresa española con independencia de la nacionalidad de quien ostente la titularidad de su capital social. De hecho, Penguin Random House Grupo Editorial es la división en lengua hispana de la multinacional alemana del sector editorial: Bertelsmann SE \& Co. KGaA, y tiene su sede social y fiscal radicada en España, concretamente en Barcelona. Es más, la empresa editorial presenta una antigüedad de casi sesenta años y fue constituida el cinco de agosto de mil novecientos cincuenta y nueve en la ciudad de Barcelona.

Tras la compra de Ediciones B y ediciones Santillana por Penguin Random House, el mapa editorial español se polariza aún más, con tres grandes grupos: Penguin Random House, Planeta y Anaya y, si tenemos en cuenta el número de empresas que 
contiene cada uno de ellos y el número de títulos que cada año inscriben en el ISBN. Hay que señalar que actualmente la facturación del sector editorial en el mercado interior supera los 2.000 millones de euros y, si se tiene en cuenta el tamaño de las empresas editoriales, se observa que las editoriales grandes generan el $61,5 \%$ de la facturación, las medianas el 25,9\% y las editoriales más pequeñas acumulan el 12,6\%.

A pesar de los avances tecnológicos y el desarrollo del comercio electrónico, el 52\% de las ventas de libros en papel se canalizan a través de librerías y cadenas de libros. Solo el $1 \%$ se comercializan a través de Internet. No obstante, la dinámica del mercado y la aparición de Internet han ocasionado que el sector se mueva hacia una distribución directa, es decir, hacia una reducción de intermediarios en las cadenas o redefinición de sus roles.

El objetivo de este artículo es analizar la evolución del sector editorial en España durante las últimas dos décadas. Con ello, se podrá comprobar hasta qué punto el sector editorial ha evolucionado y cómo ha internalizado los cambios tecnológicos que se han producido. El documento está estructurado de la siguiente manera: la sección dos analiza la relevancia del sector editorial en el marco de la economía española; la tercera sección analiza cuál es la estructura del sector editorial en España; la cuarta sección hace referencia a los cambios cualitativos que se han producido; la sección quinta presenta las nuevas tendencias; y finalmente la sección sexta resume las principales conclusiones e indica las limitaciones a las que hace frente este estudio.

\section{Relevancia del sector editorial en España}

El sector editorial español tiene un importante peso dentro de las denominadas industrias culturales, debido al volumen de negocio que genera, el número de empleos y su contribución al PIB, entre otros factores.

La industria editorial española es una de las más potentes de Europa y una de las de mayor proyección internacional. España se mantiene como una de las principales potencias editoriales del mundo, situándose como la cuarta potencia editorial del mundo (tanto en producción como en facturación), por detrás de Estados Unidos, Reino Unido y Alemania. Actualmente, casi cuarenta editoriales españolas tienen filiales en 32 países, principalmente en Iberoamérica (80\%) y en Europa (13,3\%). Por otra parte, en el ranking de las 56 editoriales más importantes del mundo por volumen de facturación, contamos con dos grupos españoles: el grupo Planeta y el grupo Santillana, en el puesto 10 y 36 , respectivamente, según los datos correspondientes al año 2016.

La industria cultural en Europa constituye un sector que se sitúa por delante de otros como, por ejemplo, la energía o la agricultura. En concreto, el sector editorial español aporta 1,1\% al PIB, lo que supone más del $34 \%$ del conjunto de las actividades culturales en España. Le siguen por orden de importancia el sector Audiovisual y multimedia $(27,5 \%)$, que incluye, entre otros, las actividades de cine, vídeo, música grabada o televisión. Los restantes sectores tienen una participación inferior, Artes plásticas (12,4\%) Patrimonio, archivos y bibliotecas (9,7\%) y Artes escénicas (9,6\%). Estas cifras ponen de manifiesto el significativo peso de las actividades culturales en el conjunto de la economía española.

La industria editorial es el sector que genera la mayor riqueza dentro de las industrias culturales. Según los datos de la FGEE (2017), los ingresos totales obtenidos en 2016 por las empresas editoriales españolas ascendieron a 2.317,20 millones de euros y generaron 12.608 son empleos directos. A esta cifra habría que añadir el empleo 
vinculado al resto de actividades asociadas a la cadena del libro. El sector editorial es, de hecho, una importante fuente generadora de empleo: en 2015, en España, el empleo cultural en su conjunto proporcionó 515.000 puestos de trabajo, un 2,9\% del empleo total en España. El empleo directo en las empresas editoriales fue incrementándose año a año hasta 2008 año en el que -por efecto de la Gran Crisis-comienza a decrecer hasta el 2015, momento en el que parece confirmarse una ligera tendencia ascendente de creación de empleo por parte de las empresas editoras.

La fortaleza del sector editorial español está permitiendo a esta industria mantener en los últimos años un saldo comercial con resultados positivos, lo que supone un elemento fundamental para el equilibrio de la Balanza Exterior. La facturación proveniente de la exportación de libros rozó en 2016 los 347 millones de euros, con México como mercado principal. Esta cifra sitúa a España, junto a Reino Unido y Estados Unidos, como uno de los tres primeros países del mundo en exportación de libros.

Europa y América siguen siendo los principales mercados exteriores del sector del libro español. Las exportaciones europeas crecieron un 4,16\% en 2015 hasta los 347 millones de euros. En cuanto a las destinadas a América, también tuvieron un incremento del 1,9\%, alcanzando los 186 millones de euros.

Hay que señalar, no obstante, que una parte de las ventas de editoriales españolas en América se hacen a través de sus filiales en países iberoamericanos y que, por lo tanto, no computan como exportaciones. La internacionalización de las editoriales españolas se vio impulsada inicialmente por la búsqueda de nuevos mercados y por las ventajas lingüísticas y culturales.

En conclusión, dentro del campo de las industrias culturales, el sector editorial ocupa un lugar clave gracias al volumen de negocio que genera, el número empleos que ofrece y las posibilidades de crecimiento y desarrollo que pueden darse como consecuencia de las innovaciones y cambios tecnológicos a los que se enfrenta el sector.

\section{Estructura del sector editorial español}

En el sector editorial español se encuentran negocios muy diferentes en dimensión (tanto en tamaño como en facturación), especialización productiva, así como muy distintas en cuanto a las condiciones de trabajo y el modelo de relaciones laborales, coexistiendo grandes grupos multinacionales hasta pequeñas empresas de producción casi artesanal.

En lo relativo a la participación en el mercado por tamaños se observa que: a) las pequeñas editoriales solo representaban el 37\% de la estructura editorial en 1996 mientras que, dos décadas después, suponen el $75 \%$ del total, b) las editoriales medianas solo han perdido un punto porcentual, pasando del 11,7\%, en 1996, al 10,9\%, en 2016, c) las editoriales grandes se mantienen muy estables, en torno al $3 \%$ y d) solo el $26,7 \%$ del total de editoriales pertenece a algún grupo empresarial.

Durante el año 2016 la producción editorial fue desarrollada por 3.026 agentes editores. Sin embargo, durante estos últimos veinte años el abandono de la actividad editorial es muy superior a las nuevas incorporaciones. Esto es debido a que los cambios tecnológicos están desafiando los modelos de negocio existentes hasta el momento e impulsa a las empresas a reexaminar sus carteras de productos y sus competencias básicas (Øiestad y Bugge 2014), lo que implica que muchas de las empresas decidan irse del mercado y no readaptarse a los nuevos cambios. 


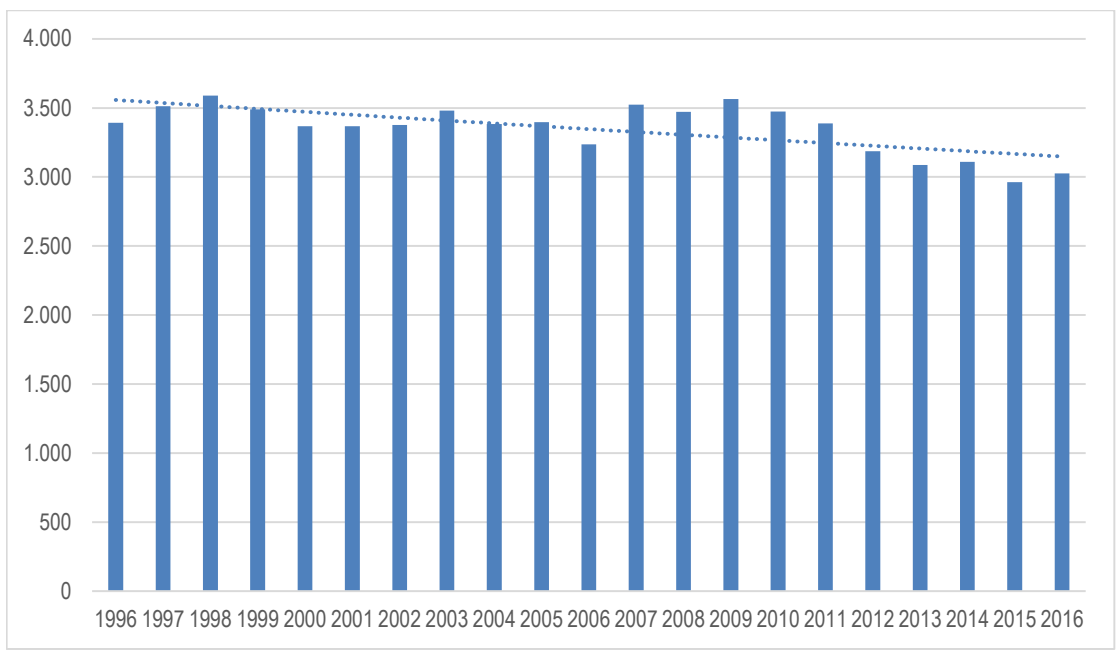

Elaboración propia a partir de los datos publicados en la Panorámica de la Edición Española (Ministerio de Cultura y Observatorio del Libro en España).

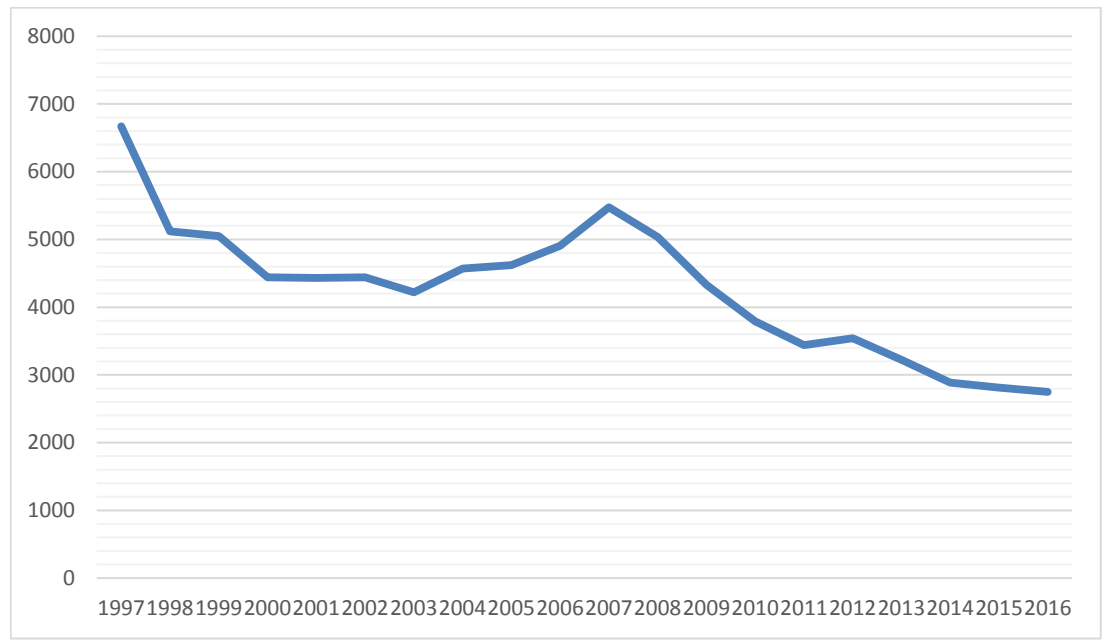

Elaboración propia a partir de los datos publicados en la Panorámica de la Edición Española (Ministerio de Cultura y Observatorio del Libro en España).

Tal y como se muestra en la Figura 1, desde el año 1996, hay en España un $9 \%$ menos de empresas editoras, una tendencia que parece ser se va a seguir manteniendo.

En la Figura 2 se ofrece la evolución anual de la tirada media de ejemplares por título publicados en España de 1997 a 2016 (en miles de ejemplares) que, como se puede observar, ha ido disminuyendo progresivamente: en 2016 la tirada media ha sido de 2.740 ejemplares, mientras que, en 1997, la tirada media se situaba en los 6.670 ejemplares, lo que ha supuesto una reducción de la tirada media en un 59\% para el período considerado. Sin embargo, se da la circunstancia de que, en el mercado editorial español, coexisten obras con fuertes tiradas y libros que no alcanzan los 1.000 ejemplares.

Analizando los datos de la Agencia Española ISBN, hay que señalar, que esta registró, por primera vez en el año 2000 , títulos en la modalidad de archivo de Internet, pero
Figura 1. Evolución de número de agentes editores (1996-2015)

Figura 2. Evolución anual de la tirada media de ejemplares por título publicados en España (1997-2016) 
Figura 3 Evolución anual de los ISBNs concedidos según soportes de publicación (1999-2016)

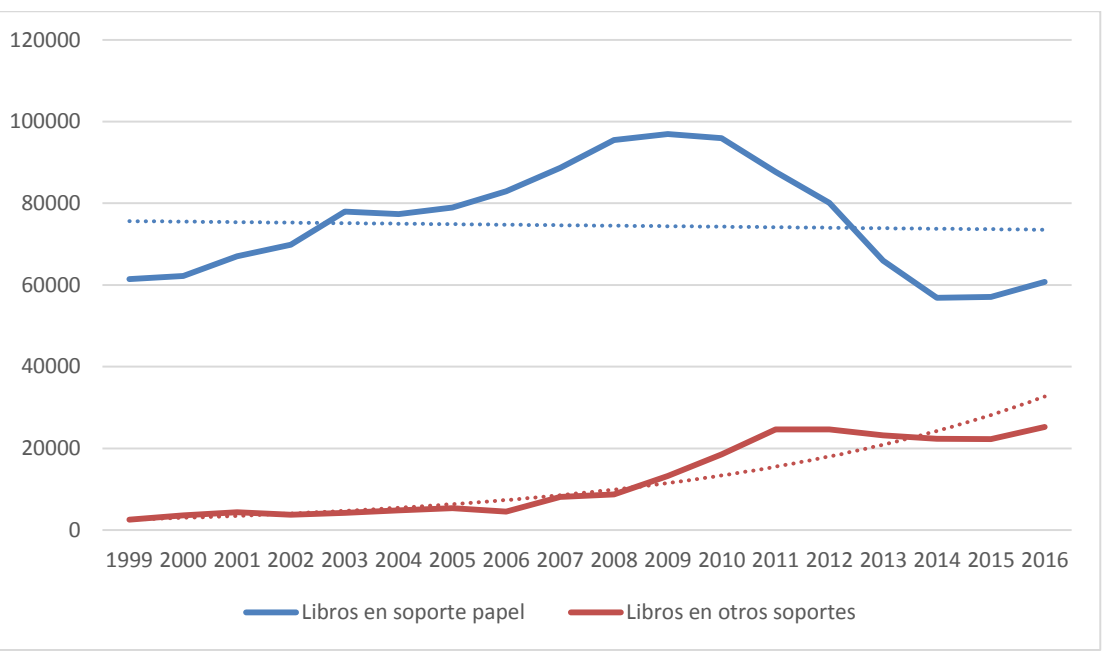

Elaboración propia a partir de los datos publicados en la Panorámica de la Edición Española (Ministerio de Cultura y Observatorio del Libro en España).

es a partir de 2012 cuando las tecnologías irrumpen con fuerza, con la utilización de los nuevos formatos PDF, ePub, Mobi (Kindle) y otros ebooks que han sustituido a los archivos de Internet provocando que, desde del año 2005, el número de ISBNs inscritos de archivos digitales, no ha parado de crecer, llegándose en el año 2010, a incrementar las cifras del año anterior en un $155 \%$.

En la Figura 3 se muestra la evolución de ISBNs concedidos según soportes "papel y otros" desde el año 1999, que es el primer año del que se dispone de datos para otros soportes que no son papel.

No será hasta el 2008 cuando la producción en libro en papel comience a caer mientras que, al tiempo, la producción de libros en otros soportes, como el libro electrónico, aumente.

En el año 2016 se publicaron en España 25.237 títulos en formato digital, incrementándose un $13,3 \%$ respecto al año anterior. De todos modos, más del $85 \%$ de los editores españoles publicaron también libros en papel, lo que conduce a la siguiente conclusión: se está produciendo una dualidad en la labor editorial, puesto que el mismo título se publica en ambos formatos.

La Figura 4 presenta la evolución anual del valor total de las exportaciones de la industria del libro de España entre 2000 y 2016, incluyendo tanto las exportaciones del sector editorial como las del sector gráfico.

En 2016, las exportaciones españolas del sector del libro alcanzaron un valor de aproximadamente 571,8 millones de euros, lo que supuso un incremento de más de 19 millones con respecto a la cifra registrada el año anterior. De ellos, el 95,8\% los han exportado las editoriales españolas directamente o a través de distribuidoras pertenecientes a sus grupos editoriales. El resto lo han hecho distribuidores independientes y libreros.

Desde el punto de vista de la exportación, el decenio 2007-2016 se caracteriza principalmente por la consolidación de la Unión Europea como mercado preferente de las exportaciones del sector, esto se debe a una apuesta por la internacionalización 


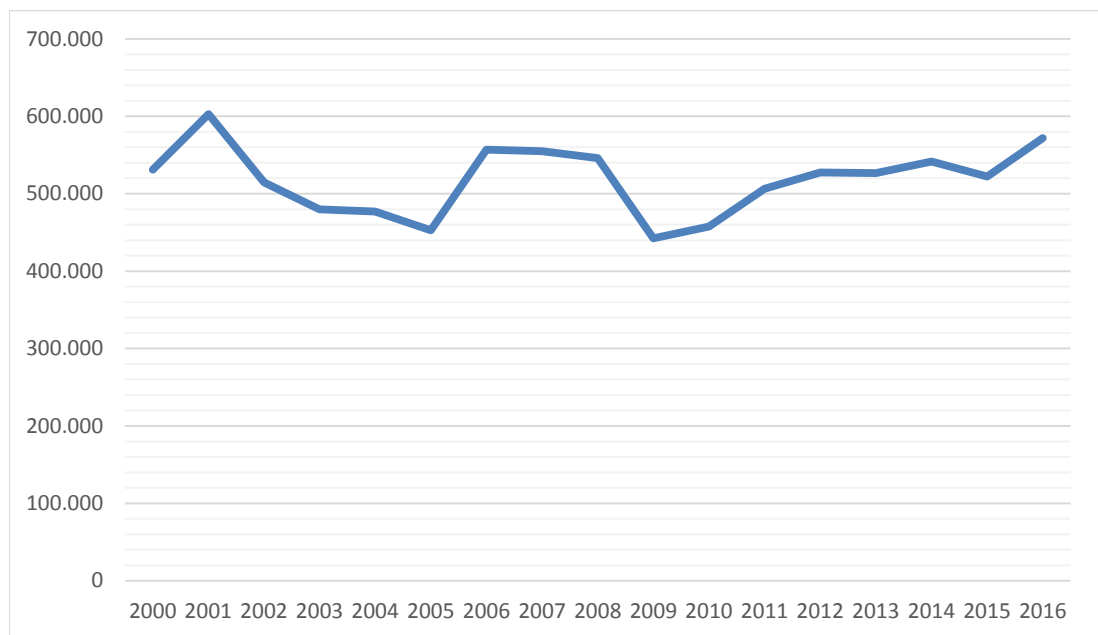

Elaboración propia a partir de los datos publicados en el Comercio Exterior del Libro (Fedecali).

de las empresas editoriales y por la crisis del sector gráfico debida a la competencia de terceros países que producen a costes más bajos.

Los factores que influyeron en la caída entre 2002 y 2005 fueron diversos y se produjeron de forma paulatina: la crisis mundial tras los ataques a las Torres Gemelas, el fortalecimiento del euro, la casi desaparición del mercado argentino y el hecho de que un buen número de las editoriales españolas comenzaron a editar e imprimir en Iberoamérica. En 2009 se produjo una fuerte contracción de la exportación de libros del sector editorial como consecuencia de la crisis económica mundial, reduciéndose de forma notable su actividad exportadora lo que significó un descenso del 18,98\% en las cifras de exportación. A partir de 2010 se produce un cambio de tendencia que se mantiene.

Se concluye que la actividad editorial en España muestra una serie de tendencias que se están asentando en los últimos años. En especial, cabe destacar: a) la existencia de más títulos, pero también de menos copias - es decir, la bajada de la tirada media-; b) el descenso de la edición impresa, como consecuencia de la creciente apuesta por la edición digital; c) aumento de la exportación; d) reducción de los agentes editores tanto públicos como privados y e) reducción del número de ejemplares vendidos.

Por tanto, la aparición y popularización del libro electrónico, la lectura en soportes digitales, el surgimiento de plataformas de autopublicación y distribución, la posibilidad de realizar tiradas cortas con la impresión bajo demanda o el nacimiento de editoriales exclusivamente electrónicas (Carreiro, 2010) están facilitando las bases para una reorganización que todavía no ha finalizado.

\section{Cambios cualitativos en el sector editorial español}

El sector editorial español no se podría entender, actualmente, sin conectar la innovación de proceso y de producto. Por un lado, se desarrollan nuevos productos, como el libro electrónico, pero, por otro lado, se mejoran los procesos de producción del libro -tanto en papel como electrónico-, al que también se aportan nuevas características, como puede ser la realidad aumentada y la impresión bajo 
Figura 5. Evolución anual del número de libros electrónicos publicados en España (2007-2017)

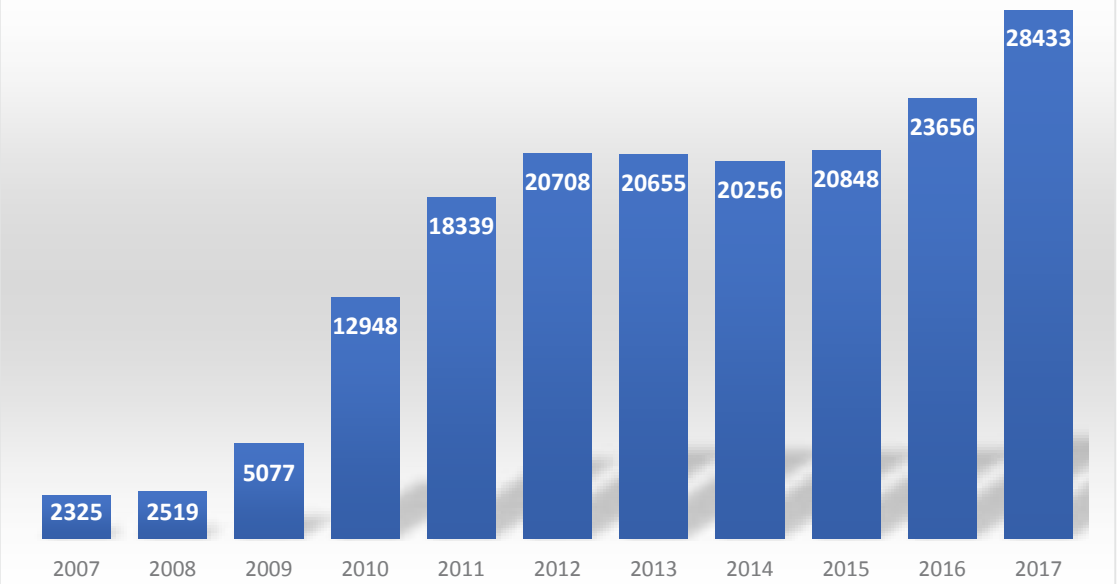

Elaboración propia a partir de la Panorámica de la Edición Española de Libros (Ministerio de Cultura y Observatorio del Libro en España).

demanda, integrando, así, el diseño y las tecnologías de fabricación (Ettlie, 1995, Ettlie y Stoll, 1990; Kim et al., 1992; Magadán, 2017).

\subsection{Libro electrónico}

El libro como medio de comunicación cuyo soporte tradicional ha sido el papel, se encuentra actualmente ante una encrucijada provocada por la disrupción del nuevo soporte electrónico, el ebook, lo que implica la mayor transformación de la industria editorial desde Gutenberg y la aparición de la imprenta (Carreiro, 2010), desafiando los modelos de negocio existentes hasta el momento en la industria editorial e impulsando a las organizaciones a reexaminar tanto sus carteras de productos como sus competencias básicas.

El libro electrónico abre la posibilidad a nuevos mercados y aplicaciones potenciales (Dess y Beard, 1984; Dewar y Dutton, 1986; Ettlie et al., 1984), lo que fuerza a empresas editoriales a replantearse (cuestionar) las formas y maneras de hacer hasta el momento para sacar provecho de las nuevas formas, que conducen, por ejemplo, a nuevas capacidades/habilidades tecnológicas y/o comerciales o a emplear nuevos modos o métodos de solución de problemas (Dewar y Dutton, 1986; Ettlie et al., 1984; Henderson y Clark, 1990; Tushman y Anderson, 1986).

Con independencia de sus cualidades como producto, el libro electrónico ha cambiado el modo de leer, la manera en que un lector puede aproximarse a un texto. En el caso de España, la edición electrónica experimentó en los últimos años un aumento significativo principalmente en el segmento de archivos de Internet (Cordón et al., 2010). Será con el lanzamiento del Kindle de Amazon y del iPad de Apple cuando comience a desarrollarse la distribución y comercialización del libro electrónico (Clark et al., 2008; Wischenbart, 2016), lo que sugiere que uno de los factores que había limitado el desarrollo de la industria editorial digital fue la falta de un lector electrónico suficientemente sofisticado como para atraer a los lectores al ámbito digital (Gaigher, 2012).

El nivel de desarrollo del mercado del libro digital en España sigue una línea similar a la de otros muchos países de nuestro entorno, con la excepción de -en el ámbito europeo-Reino Unido, país que se sitúa a la cabeza por detrás de Estados Unidos. En el caso de España, la edición electrónica experimentó en los últimos años un aumento 


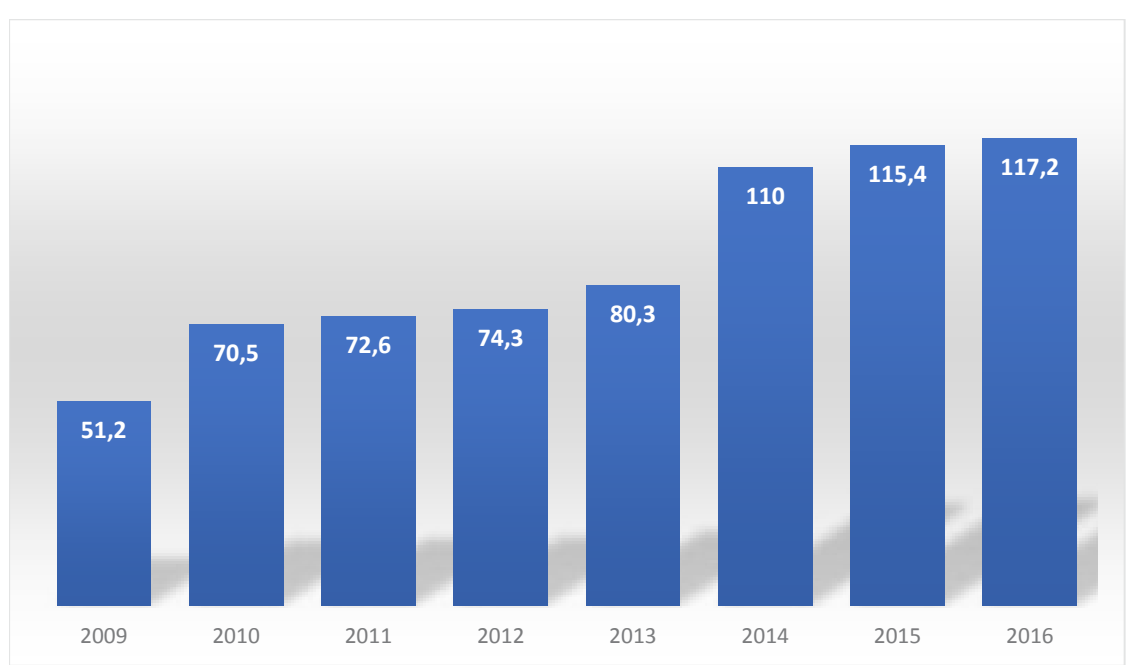

Elaboración propia a partir de El Comercio Interior del Libro (FGEE).

significativo. En la Figura 5 se muestra la evolución anual del número de libros electrónicos publicados en España entre 2007 y 2017. En el año 2017 se registraron en España 28.433 libros digitales, que representa casi el 30\% de producción editorial, mientras que el 2008, por ejemplo, solo suponía el 2,4\%. En 2016, se editaron 23.653 libros digitales, lo que supuso un incremento de alrededor de 2.800 ejemplares con respecto a la cifra registrada el año anterior.

Además de la innovación de producto que ha supuesto el ebook, en los últimos años se están introduciendo en el sector otras innovaciones de producto (Wischenbart, 2016) como: a) el audiolibro y b) el libro puente. En cuanto a la mejora de las características de tales productos editoriales, nos encontramos con: a) la realidad aumentada, en el libro impreso y electrónico y b) los códigos QR, en el libro impreso.

Desde 2010 el número de empresas editoriales que editan en formato electrónico ha aumentado un $70 \%$. Estos datos muestran que, en estos últimos años, las organizaciones editoriales buscan nuevos soportes y apuestan por ellos. Surgen editoriales que solo editan libro digital, y muchas de ellas editan en ambos formatos.

En 2016, el 87\% de los títulos editados en formato digital se publicaron también en papel, y solo el $13 \%$ se editaron únicamente en formato digital.

A pesar del incremento en la producción de libros digitales su facturación continúa siendo residual, no supone más del $5 \%$ del total facturado en el sector durante el ejercicio, la cual se canaliza mayoritariamente $(79,5 \%)$ a través de plataformas y distribuidoras digitales, como Amazon (con una cuota del 22,6\% en 2016), Casa del Libro, Google o Apple Store, entre otras.

En cualquier caso, el peso de la facturación por libros digitales sobre el total facturado en cada materia continúa siendo bastante reducido en comparación con el resto de soportes con la única excepción de los libros de Derecho y Ciencias Económicas -incluidos en la categoría Ciencias Sociales y Humanas. En la Figura 6 se muestra la cifra de negocio de la industria de los libros editados en formato electrónico en España desde 2009 hasta 2016, expresada en millones de euros lo que significó un incremento de aproximadamente dos millones con respecto a la cifra registrada el año anterior. La venta de libros en formato electrónico ha facturado 117 millones de
Figura 6. Evolución anual de la cifra de negocio de la industria de los libros editados en formato electrónico en España (20092016), en millones de euros. 
euros en 2016, un 1,5\% más que en 2015. Aunque la facturación generada a través de las ventas de libro electrónico sigue en ascenso, esta no supone más del $5 \%$ de la facturación total de las empresas editoriales.

$\mathrm{Al}$ igual que en el formato papel, las editoriales españolas exportan mucho contenido digital: el $60 \%$ de las descargas digitales de los libros españoles se realizan fuera de España, principalmente en América Latina y Estados Unidos consolidando la tendencia exportadora de la producción de las editoriales españolas.

A lo largo del año 2016, el 53\% de las ventas de ebooks de las editoriales españolas tuvo lugar en España, mientras que el resto de las ventas tuvo lugar en América Latina (33\%), el mercado hispano de Estados Unidos (9\%), Europa (4\%) y el resto del mundo (1\%).

En la distribución de los libros digitales la mayoría de los nuevos jugadores provienen de la industria tecnológica y tienen pocos vínculos con el mundo de la edición (Magadán, 2017). Los fabricantes de hardware y proveedores de servicios de Internet han entrado en la industria del libro y pueden superar a los actores tradicionales de la industria en la creación de nuevos modelos de negocio para el contenido digital.

\subsection{Edición bajo demanda}

La irrupción de la impresión digital ha dotado a los talleres de artes gráficas de herramientas mucho más ágiles - principalmente en tiempo- para dar respuesta a las nuevas demandas: tiradas cortas, impresión bajo demanda -cada vez más frecuente- y personalización de los ejemplares que está modificando la forma de producir el libro impreso (McAllister et al., 2002; Fabling 2017).

La edición bajo demanda (en adelante, POD) está transformando la forma en que los editores ofertan los libros en papel, esto es, ya no es necesario imprimir varias unidades de un título y esperar a que se vendan (Sánchez Tarragó y Díaz Álvarez, 2005).

Con este sistema se puede ofrecer el título en una librería o plataforma, si se produce un pedido entonces se procede a su impresión y posterior envío al cliente (Gallagher, 2014; Magadán, 2017). De manera que el editor no ha tenido que hacer una inversión en su producción, reduciendo su riesgo empresarial y, además, no necesita almacenarlos evitándose las devoluciones por parte de las librerías y distribuidores (Senftleben et al., 2017).

Muchas plataformas, como Amazon, han puesto en marcha la impresión bajo pedido, combinándose impresión, distribución y venta desde su web a cualquier parte del mundo. La POD ha reducido las barreras a la entrada en el mercado y ha proporcionado una solución para las editoriales pequeñas para poder acceder a un mercado global vinculándose a un mayorista o minorista que tiene capacidad para realizar POD.

A diferencia de lo que significan las producciones de tiradas muy cortas (de 50 a 100 ejemplares), la impresión bajo demanda permite la producción de un libro como una respuesta específica a una orden del cliente sin necesidad de formar parte de ningún stock. Del mismo modo, al facilitar la reimpresión "a la carta", los títulos no están agotados nunca. Con un modelo que supone vender primero el ejemplar y después producirlo (proceso inverso al tradicional), cualquier editorial puede ofrecer un título tanto en su sitio web como en otras plataformas de distribución y venta globales.

En España la edición bajo demanda solo representa el 0,5\% del total editado en soporte papel en España. Esto es debido a que muchos editores solo lo emplean como 
alternativa para la reimpresión de títulos descatalogados. Este modelo de impresión 1:1 podría servir para garantizar la reposición y la venta por goteo, principalmente en editoriales de tamaño mediano y pequeño. A los editores españoles les preocupa mucho la calidad de un libro impreso de manera digital (Magadán, 2017).

El cambio tecnológico que esta experimentado el sector editorial español está dando lugar a que se desarrollen nuevos modelos de negocio que surgen de la adaptación a sus estructuras productivas de aquellas innovaciones que han ido surgiendo a partir de las tecnologías de la información y la comunicación.

Por ejemplo, Logista Libros, el mayor distribuidor independiente de libros en España, ha lanzado recientemente un nuevo servicio integrado de impresión bajo demanda y distribución en colaboración con Lantia Publishing, líder en la aplicación de las tecnologías de la información en el sector editorial.

En 2016, Confederación Española de Gremios y Asociaciones de Libreros (CEGAL) incorpora la impresión bajo demanda en su alianza con Podiprint para que las librerías de toda España puedan incorporar en sus catálogos más de 5.000 títulos hasta el momento inaccesibles para las librerías.

\section{Nuevos modelos de negocio en la industria editorial española}

Los modelos de negocio deben construirse sobre un plan lógico para llevar un producto al mercado y obtener un beneficio. En este sentido, las empresas tienen que innovar en sus respectivos modelos de negocio con nuevas formas de crear y capturar valor para sus grupos de interés (Afuah y Tucci, 2002; Zott y Amit, 2007).

El modelo de negocio no solo proporciona la arquitectura lógica detrás de la producción y el suministro de un producto, sino que, además, aporta una comprensión y respuesta por parte de la empresa al mercado y sus necesidades (Morris, 2009; McGrath, 2010; Cavalcante et al., 2011; Øiestad y Bugge, 2014).

La digitalización en el sector editorial ofrece nuevas formas de presentar el contenido (Shatzkin, 2008; Magadán, 2017) y permite que se puedan desarrollar nuevos modelos de negocio (Hoque, 2000, Gordon et al., 2008; Sabatier y Fizelle, 2011; Peng, 2016), adoptando las innovaciones surgidas al amparo de las tecnologías de la información y la comunicación (Rayport y Sviokla, 1995; Amit y Zott, 2001; Porter, 2001) con objeto de ampliar su cartera de productos (Rayna y Striukova, 2016).

No se puede negar que el cambio en el modelo de negocio existe, aunque el libro impreso sigue siendo, por el momento, el producto más rentable dentro de la cartera de productos editoriales. Entre los posibles modelos de negocio que las empresas editoriales españolas están ensayando actualmente, se puede destacar: el contenido fragmentado, el pago por consumo o contenido a la carta, el modelo de suscripción, la afiliación, el crowfunding, la gamificación, la venta directa y la autoedición (Magadán, 2017).

La venta de contenidos fragmentados en Internet se inició con la música, a través de Itunes, que ofrecía canciones sueltas al margen del disco original. Esa fragmentación está presente en toda la industria cultural, incluido el ámbito editorial. En España, el sello Gestión 2000 del Grupo Planeta fue pionero al vender en capítulos sueltos de libros y Random House Mondadori creó, dentro del sello editorial Debate, una colección denominada enDebate para publicar textos breves de no ficción de unas 10.000 palabras en formato digital. 
El pago por consumo o contenido a la carta está basado en ofrecer al cliente la posibilidad de pagar por lo que realmente consume. Se diferencia del contenido fragmentado en que puede que no sea una parte del total, sino que se trate del contenido completo. A raíz de este tipo de iniciativas y conscientes de la demanda existente - sobre todo en libros de no ficción por la consulta y adquisición de capítulos o partes del mismo-, surgió Slicebooks. El grupo Planeta ha lanzado la iniciativa Planetahipermedia.com, una web de formación empresarial a través vídeos y materiales textuales cortos sobre un tema específico.

La suscripción es un modelo adoptado de forma mayoritaria porque conlleva unas ventajas muy interesantes desde el punto de vista empresarial, entre ellas, contar con una base de datos con información de nuestros clientes y con un flujo de ingresos fijo y preestablecido, que permite adecuar la oferta a la demanda que se tiene, por lo que es positivo también para la planificación del negocio. Los modelos de suscripción llegaron al sector editorial a través de las suscripciones de contenidos de índole jurídica y con libros técnicos. Hoy en día, existen diferentes plataformas generalistas en España que ofrecen este servicio para el lector como 24Symbols y Nubico. En el mercado digital español, la empresa pionera en la introducción de este modelo fue 24Symbols. Eso sí, diez años más tarde, en 2011. A esto, añadió la lectura en streaming desde cualquier dispositivo con acceso a un navegador, apoyándose en el cloud computing. Aunque las ventas derivadas de las librerías online como Amazon, Apple, Kobo, entre otras, siguen siendo uno de los canales prioritarios de ventas para las editoriales españolas, las ventas derivadas de las plataformas de subscripción en 2017, especialmente en Estados Unidos, Alemania, España y México, se están volviendo cada año más relevantes para las editoriales alcanzando el $6 \%$ de las ventas digitales totales en comparación con el $5 \%$ que representaba en 2016. La venta de licencias de libros electrónicos a las bibliotecas alcanzó el 3\% del total de las ventas digitales en 2017 en comparación con el $2 \%$ alcanzado en 2016. Esto significa que el modelo de suscripción se va asentando.

La afiliación consiste en ser miembro o socio de un grupo o servicio, lo que implica en nuestro análisis pertenecer a un grupo de clientes que, en términos de intercambio, se vincula a un conjunto de servicios y contenidos ofrecidos por una empresa, cuya afiliación puede conllevar o no un pago económico. Dentro de este modelo puede que se incluyan otros, como el pago por consumo, o, lo que es más habitual, la suscripción a ciertos servicios o contenidos. No obstante, en principio, son dos modelos diferentes, y aunque están próximos, no hay que confundirlos y conviene considerarlos por separado. En el mundo editorial español, Círculo de Lectores es el referente al utilizar este modelo de negocio en el formato papel desde 1962 y continuar con él cuando en 2010 fue adquirido por el Grupo Planeta. Pertenecer a dicho club no tiene ningún coste, pero existe la obligación de permanecer al menos dos años y durante ese tiempo adquirir al menos un producto sin importar el valor del mismo de cada una de las siete revistas en papel que se reciben al año. A cambio de pertenecer a este grupo, el usuario recibe ofertas especiales de hasta el 30\% no solo en libros, sino en otros servicios de cultura, ocio o cualquier sector, que pueden negociarse o conseguirse gracias al volumen de socios, que en la actualidad supera el millón.

La esencia del crowdfunding es el micromecenazgo multitudinario, que permite abordar proyectos, al margen de los cauces de financiación convencional, a cambio, en muchos casos, de una participación en los beneficios derivados del proyecto o en un acceso gratuito o más ventajoso al servicio o producto derivado del proyecto, como consecuencia de la condición de micromecenas. En España la plataforma Libros.com ha basado su negocio en el crowdfunding, por lo que se trata de una editorial que asegura una edición, diseño y distribución de calidad sin ningún coste para el autor. 
El concepto de gamificación o ludificación tiene que ver con el uso del juego en contexto ajenos al puro entretenimiento -de hecho, también se les denomina juegos serios- con la intención, por ejemplo, de adquirir destrezas y competencias en áreas como la educación o el desarrollo profesional, entre otras. Una de las primeras orientaciones de la gamificación en el sector editorial ha sido la del desarrollo del marketing relacional, que busca incrementar la participación activa en las redes sociales empresariales de los potenciales clientes que navegan por Internet.

La venta directa sigue en auge como otra posible vía de ingresos complementaria del modelo tradicional de venta a través de terceros y consiste en la comercialización y venta de productos directamente a los consumidores sin ningún tipo de intermediario o distribuidor (modelo $\mathrm{B} 2 \mathrm{C}$ ). La venta directa por Internet no llega al 1\% de la facturación en las empresas editoriales, pero les puede facilitar la integración de todos los procesos de venta $\mathrm{B} 2 \mathrm{~B}$ - ajenos al canal de distribución tradicional- a través de sus plataformas web, ofreciendo mejores precios y servicios más personalizados: sugerencias partiendo del historial individual de compras o visionado, descuentos en contenidos relacionados con los intereses del usuario, entre otros. El punto fuerte de la venta directa estriba en el conocimiento directo del cliente y de su comportamiento a lo largo del proceso de compra, a lo que se añade la posibilidad de análisis sus pautas de consumo. En este campo, Bookmobile facilita a las editoriales poder crear y gestionar tiendas o plataformas para la venta directa sin necesidad de terceros. Dentro de estas oportunidades hay otros casos, como el de Pubsoft, que ofrece el software necesario para la gestión de todos los procesos de promoción y venta de contenidos online. Con esta plataforma en la nube, los editores y autores son capaces de administrar autónomamente cuentas de autor, cargar ebooks, vender directamente a los lectores.

Los editores pueden convertirse en sus propios libreros a través de la web, mientras que los autores pueden ayudar a los editores a comercializar sus títulos en línea: por ejemplo, a través de las redes sociales (Hyatt, 2003).

El principal canal para la distribución y venta de libros en formato electrónico en España son plataformas específicas de distribución digital (79,5\%), especialmente a través de plataformas comerciales genéricas como son Amazon, Google Play, Casa del Libro, App store, entre otras siendo las plataformas específicas de distribución el medio que condensa mayor facturación.

La venta directa desde la web de la editorial gana peso y supone $19 \%$ de la facturación. Por su parte, la venta a través de librerías pierde terreno solo representa el $1,5 \%$. Inherente a este escenario es, también, el hecho de que los editores pierden el control sobre el contenido; e incluso pueden llegar a ser un eslabón cada vez menos necesario si los autores optan por asumir todo el proceso de publicación en el nuevo contexto digital (Gaigher, 2012).

En la elaboración y distribución de ebooks, la mayoría de las editoriales se ven compelidas a interactuar principalmente con empresas del sector tecnológico -sin apenas vínculos con el mundo de la edición- para abordar los problemas y desafíos derivados del cambio tecnológicos y sociocultural en el que están inmersas (Loebbecke, 2010; Longhi y Rochhia, 2014; Protogerou et al., 2017).

En lo que respecta a los dispositivos de lectura para los que se comercializa la obra digital, de acuerdo con Comercio Interior del Libro en España, el ordenador ha perdido sustancialmente terreno respecto a años anteriores, descendiendo un $24,9 \%$. Los resultados muestran una mayor tendencia hacia la comercialización de contenidos para ereaders -el $31,8 \%$ de las obras digitales se han comercializado para su lectura 
en estos dispositivos-y tabletas (5,2\%), aunque también se incrementa la venta de contenidos para su lectura en streaming $(4,4 \%)$.

En los últimos años, editoriales y librerías apuestan firmemente por hacerse un espacio en el mundo de la autoedición, como una línea más de su negocio. Las plataformas de autoedición vinculadas a los sellos editoriales podrían redefinir los límites, hasta ahora establecidos, entre escritores y aspirantes a escritores (autores indies) en el mundo del libro.

En España Bubok es una plataforma pionera de autoedición online que permite editar, publicar y vender libros bajo demanda, tanto en formato papel como digital. Asimismo, Bubok también es una tienda de libros online y offline, ya que recientemente acaba de abrir una tienda física en Madrid.

Book Country de Penguin Random House ha diseñado un espacio en el que descubrir libros y autores nuevos, discutir, compartir consejos y experiencias y aprender sobre la industria editorial. En España, el mismo grupo editorial ha creado Me gusta escribir, una red social para escritores donde, además de incluir recursos para escritores, consejos o noticias, ofrecen servicios de autopublicación que van desde el diseño o la revisión ortotipográfica a la promoción y el marketing.

El grupo Planeta acaba de lanzar en marzo de 2017 el Universo de Letras, una nueva plataforma de autoedición profesional que nace para dar servicio a quienes desean cumplir el sueño de ver publicado su libro. Además, funcionará como plataforma de observación que fomente el descubrimiento de nuevos autores, por lo que aquellas obras que destaquen por su difusión serán valoradas para su posible publicación bajo aquel sello editorial del Grupo Planeta que mejor se ajuste a las características del título.

Algunas cadenas de librerías también han apostado por la autoedición, como Casa del Libro a través de Tagus, una plataforma que permite a los usuarios publicar sus libros de manera independiente, pero ofreciendo las prestaciones y la calidad de una editorial tradicional. Los libros que más éxito tienen en esta fase son seleccionados por Ediciones Tagus, que realiza una nueva edición profesional del libro, acciones promocionales, e incluso puede que llegue a realizar una tirada en papel.

\section{Conclusiones}

Los cambios tecnológicos y las innovaciones juegan un papel clave en la estructura actual del sector editorial. Los editores españoles están adaptando su estrategia editorial a las nuevas necesidades de demanda digital y los nuevos dispositivos tecnológicos emergentes. En estos momentos, los viejos modelos de negocio conviven con los nuevos modelos que surgen. Sin embargo, algunas editoriales no se adaptan al cambio tecnológico o lo hacen a un ritmo más lento de lo que sería deseable. Principalmente en estos últimos cinco años, las editoriales españolas han ido adquiriendo conocimientos digitales y han intentado posicionarse para poder responder a los cambios del mercado y las tecnologías cambiantes, pero los productos digitales aún son considerados secundarios al libro en papel. De hecho, los sistemas de distribución desarrollados en torno al libro electrónico, como la plataforma Libranda, replican la cadena tradicional del libro en papel.

A la luz de las cifras del sector editorial en España se puede señalar que el desarrollo de Internet y las tecnologías de la información está modificando los modelos de negocio y provocando cambios organizativos en las empresas del sector editorial. 
El cambio tecnológico modifica la forma de producir el contenido y su distribución, afectando a la cadena de valor del sector editorial.

A pesar la progresiva evolución del peso de la edición digital sobre el global de la producción, la proporción de títulos disponibles en formatos digitales sigue siendo reducida en relación al número de títulos publicados en papel. En este sentido, puede deberse: a) falta de recursos económicos y de personal especializado que limita la edición digital, b) cierta aversión al riesgo o miedo a la piratería, que las apuntala en el modelo de negocio tradicional predominante del papel, c) elevada incertidumbre asociada a los aspectos técnicos (formatos y procesos dominantes), y d) falta de apoyo institucional que estimule su transición digital y la redefinición de sus respectivos modelos de negocio.

Las potencialidades que generan las tecnologías de la información y de la comunicación repercuten también en la necesidad urgente de revisar las propias normas de regulación de los mercados de la edición, particularmente las normas de protección de los derechos de autor, para salvaguardar la propiedad intelectual de las creaciones que se difunden por los diversos canales actualmente al alcance, tanto en soporte papel como en soporte electrónico. Habrá que reformular los contratos de edición para adaptarlos a las nuevas necesidades y modelos de negocio. La economía digital ha complicado el contexto en el que se mueve la empresa editorial lo que ha provocado un efecto disruptivo sobre las empresas editoriales y sus modelos de negocio.

Las innovaciones que se han producido en el sector editorial fueron adoptadas de modo progresivo y con cautela por la industria ante las reticencias derivadas de la observación de otros sectores, como la música o el cine, y análisis de la experiencia destilada en otros países. El libro electrónico, no acaba de convertirse en una alternativa comercialmente más rentable que el libro tradicional, pero, sin embargo, ninguna de las empresas del sector desea quedar descolgada de esta innovación. Incluso, comienzan a surgir editoriales exclusivamente orientadas a la producción de libro electrónico. A esas reticencias o cautelas de la industria, le sigue un interés por parte de los autores de seguir viendo su obra publicada en papel y una clara actitud de los lectores por continuar apostando por el libro tradicional en lugar de asumir la adquisición de un dispositivo de lectura de libros digitales. El libro digital, más que verse como un sustitutivo del libro tradicional, se está gestionando por las empresas del sector editorial como un complementario.

Por el momento, los editores españoles se están centrando en su mayoría en publicación de versiones digitales de sus libros en papel en las plataformas de distribución digital, en un intento de minimizar riesgos al apostar por obras contrastadas positivamente en el modelo de negocio tradicional. Esta decisión estratégica conduce, al menos en el corto plazo, a que sean las empresas tecnológicas, como Apple o Google, con suficientes recursos financieros y conocimientos especializados quienes controlen la distribución y venta del libro digital.

De todo el análisis realizado en este estudio se pueden establecer una serie de conclusiones e implicaciones para el sector editorial español.

En primer lugar, el sector editorial español presenta dos líneas de negocio que coexisten como complementarias y no como sustitutivas: por una parte, el libro en papel y, por otra, el libro electrónico.

En segundo lugar, por el momento, las editoriales se han limitado a realizar un simple cambio de formato de libro: del papel al electrónico, y no han avanzado ni impulsado el desarrollo de todas aquellas posibilidades que las tecnologías de la información 
puedan ofrecer como enlaces a webs, hipervínculos e imágenes, entre otros. Es necesario que las editoriales no deban limitarse a realizar un simple cambio de formato de libro (del papel al electrónico): deberían impulsar el desarrollo de todas aquellas posibilidades que las tecnologías puedan ofrecer, liderando la transición digital en la industria del libro.

En tercer lugar, la falta de iniciativas digitales conjuntas está provocando que nuevos actores ajenos al sector, como Amazon, hayan entrado en la industria del libro y puedan superar a los actores tradicionales (editoriales, distribuidores y librerías) de la industria en la creación de nuevos modelos de negocio para el contenido digital. Las editoriales consideran que han asumido ya tantos riesgos comerciales y estéticos al crear nuevos contenidos que no pueden afrontar riesgos técnicos adicionales. Como consecuencia, son reacios a invertir directamente en proyectos de I+D y prefieren adoptar soluciones técnicas contrastadas. Este proceso está dando lugar una dependencia involuntaria de las innovaciones externas que determinen la evolución real del sector.

Finalmente, en cuarto lugar, el desarrollo de la edición digital va a exigir a las empresas adaptarse a un nuevo entorno comercial virtual y global. El libro electrónico requiere un nuevo tratamiento del contenido -desde la producción hasta la distribución- que en la era digital pasa obligadamente por las tecnologías de la información y relaciones comerciales, marcadas, a su vez, por las plataformas de ventas virtuales y globales.

Como implicaciones derivadas de las conclusiones anteriormente expuestas para las empresas del sector editorial español se destacan: a) favorecer el emprendimiento orientado a la creación de nuevas empresas del sector que sean capaces de vincular la base tecnológica con la creatividad y el conocimiento; b) replantear su modelo de negocio ante las transformaciones y cambios operados en procesos y productos; c) apostar definitivamente - junto con las imprentas- por la impresión digital, los nuevos materiales, la venta online y desarrollar la impresión 1:1; d) repensar su modelo de negocio buscando el modo de vincular ambas realidades: el libro en papel y el libro digital. Han de ser conscientes de que lo verdaderamente relevante, lo que aporta valor añadido es el contenido, no el soporte en el que se plasma aquel; e) redefinir el nuevo papel de las distribuidoras ante los cambios operados en el canal de comercialización; f) aunar venta física y digital en los puntos de comercialización minorista y g) adaptar el marco normativo a los nuevos cambios para la adecuada protección de los derechos de autor.

Para concluir, es preciso destacar que este trabajo no deja sino de ser una muestra del pasado y presente del sector editorial español, pero supondría el primer paso para continuar investigando aspectos parciales de una manera más profunda. En este sentido, se sugieren las posibles líneas de investigación derivadas de este estudio. En primer lugar, se propone un análisis en profundidad sobre el papel del libro electrónico en el sector editorial español. En segundo lugar, se propone estudiar las razones por las cuales las editoriales de tamaño mediano y pequeño no instrumentan fórmulas para colaborar en el desarrollo de plataformas digitales. En tercer lugar, se propone estudiar los nuevos modelos de negocio surgidos en el sector editorial español. Finalmente, se propone estudiar el potencial de Internet como canal de exportación para las imprentas y editoriales españolas. 


\section{Referencias bibliográficas}

"Afuah, Allan y Christopher Tucci. 2000. Internet business model and strategies. New York: Mc Graw Hill.

" Amit, Raphael y Christoph Zott. 2001. Value creation in e-Business. En Strategic Management Journal. Vol. 22, no. 6, 493-520. https://doi.org/10.1002/smj.187

"Benghozi, Pierre-Jean y Elisa Salvador. 2016. How and where the R andD takes place in creative industries? Digital investment strategies of the book publishing sector. En Technology Analysis and Strategic Management. Vol. 28, no. 5, 568582. https://doi.org/10.1080/09537325.2015.1122184

" Bockstedt, Jesse, Robert J. Kauffman y Frederick Riggins. 2006. The move to artist-led on-line music distribution: a theory-based assessment and prospects for structural changes in the digital music market. En International Journal of Electronic Commerce. Vol. 10, no. 3, 7-38.

"Bugge, Markus. 2011. Creative distraction: lack of collective learning in adapting to online advertising in Oslo, Norway. En Industry and Innovation. Vol.18, 227-248. https://doi.org/10.108o/13662716.2011.541106

"Carolan, Simon y Christine Evain. 2013. Self-publishing: opportunities and threats in a new age of mass culture. En Publishing Research Quarterly. Vol. 29, no. 4, 285-300. https://doi.org/10.1007/s12109-013-9326-3

»Carreiro, Erin. 2010. Electronic books: how digital devices and supplementary new technologies are changing the face of the publishing industry. En Publishing Research Quarterly. Vol. 26, 219-235.

"Cavalcante, Sérgio; Peter Kesting y John Ulhøi. 2011. Business model dynamics and innovation: (re)establishing the missing linkages. En Management Decision. Vol. 49, no. 7-8, 1327-1342. https://doi.org/10.1108/00251741111163142

"Clark, Dennis; Susan Goodwin, Todd Samuelson y Catherine Coker. 2008. A qualitative assessment of the Kindle e-book reader: results from initial focus groups. En Performance Measurement and Metrics. Vol. 9, no. 2, 118-129. https:// doi.org/10.1108/14678040810906826

"Cordón, José Antonio; Julio Alonso y Helena Martín. 2010. Electronic books: the third wave of the digital revolution. En Anales de Documentación. Vol. 13, 53-80.

"Currah, Andrew. 2006. Hollywood versus the Internet: the media and entertainment industries in a digital and networked economy. En Journal of Economic Geography. Vol. 6, 439-468. https://doi.org/10.1093/jeg/lbloo6

»Dess, Gregory y Donald Beard. 1984. Dimensions of organizational task environments. En Administrative Science Quarterly. Vol. 29, 52-73. https://doi. org/10.2307/2393080

»Dewar, Robert y Jane Dutton. 1986. The adoption of radical and incremental innovations: An empirical analysis. En Management Science. Vol. 32, no. 1, 14221433. https://doi.org/10.1287/mnsc.32.11.1422

"Donoughue, Peter. 2010. Beyond the fear of cannibalisation will the book publishing industry survive the digital revolution?. En Logos. Vol. 21, no. 3, 167-178. https://doi.org/10.1163/095796511X560060 
》Ettlie, John. 1995. Product-process development integration in manufacturing. En Management Science. Vol. 41, no.7, 1224-1237. https://doi.org/10.1287/mnSc.41.7.1224

»Ettlie, John; William Bridges y Robert O’Keefe. 1984. Organization strategy and structural differences for radical versus incremental innovation. En Management Science. Vol. 30, no. 6, 682-695. https://doi.org/10.1287/mnsc.30.6.682

"Ettlie, John y Henr Stoll. 1990. Managing the design-manufacturing process. New York: McGraw-Hill College.

"Evans, David. 2008. The economics of the online advertising industry. En Review of Network Economics. Vol. 7, 359-391. https://doi.org/10.2202/1446-9022.1154

» Fabling, Timoth. 2017. Introducing remote printing into the publishing industry of a small, remote economy: The case of New Zealand. Doctoral dissertation, University of Wellington. <http://hdl.handle.net/10063/6208> [Consulta: 10 junio 2018].

» Gaigher, Susan. 2012. Digital publishing in the South African trade sub-sector: lessons to learn from disruptive technology, Doctoral dissertation, University of Pretoria. <http://hdl.handle.net/2263/32784> [Consulta: 10 junio 2018].

» Gallagher, Kell. 2014. Print-on-Demand: new models and value creation. En Publishing Research Quarterly. Vol. 30, no.2, 244-248.

»Galuszka, Patryk. 2015. Music aggregators and intermediation of the digital music market. En International Journal of Communication. Vol. 9, 254-273.

" Gordon, Linda; David Kung y Harold Dyck. 2008. Strategic use of e-commerce in the transformation of the publishing industry. En Communications of the International Information Management Association. Vol. 8, no. 4, 65-78.

"Henderson, Rebecca y Kim Clark. 1990. Architectural innovation: the reconfiguration of existing product technologies and the failure of established firms. En Administrative Science Quarterly. Vol. 35, no. 1, 9-30. https://doi. org/10.2307/2393549

" Hyatt, Shirle 2003. Judging a book by its cover: e-books, digitization and print on demand. En G. Gorman. The digital factor in library and information services. London: Facet Publishing. p. 112-132

" Ho, Hui-Yi; Luh-Wang Wang y Hsueh-Ju Cheng. 2011. Authors, publishers and readers in publishing supply chain: the contingency model of digital contents production, distribution, and consumption. En Systems Engineering Procedia. Vol. 2, 398-405. https://doi.org/10.1016/j.sepro.2011.10.053

"Hoque, Faisal. 200o. E-enterprise, business models, architecture and componets. New York: Cambridge University Press.

》Hracs, Brian. 2012. A creative industry in transition: the rise of digitally driven independent music production. En Growth and Change. Vol. 43, 442-461. https://doi.org/10.1111/j.1468-2257.2012.00593.x

» Kim, Jay; Larry Ritzman, W. C. Benton y David Snyder. 1992. Linking product planning and process design decisions. En Decision Sciences. Vol. 23, no.1, 446o. https://doi.org/10.1111/j.1540-5915.1992.tboo376.x

» Lichtenberg, James. 2011. In from the edge: the progressive evolution of publishing in the age of digital abundance. En Publishing Research Quarterly. Vol. 27, no. 2, 101-112. https://doi.org/10.1007/s12109-011-9212-9

» Loebbecke, Claudia. 2010. The emergence of ebooks: just another media industry joining the converging digital world? An explorative study on user preferences 
and industry structure changes. Washington, DC, Telecommunications Policy Research Conference.

"Longhi, Christian y Sylvie Rochhia. 2014. This will kill that? Dynamics of changes in the book industry. En Revue d'Économie Industrielle. No. 145, 121-154. <https:// journals.openedition.org/rei/5761> [Consulta: 10 junio 2018].

" Magadán, Marta. 2017. Cambio tecnológico e innovación en el sector editorial español: efectos organizativos. Tesis doctoral, Universidad de Oviedo. <http://hdl. handle.net/10651/44590> [Consulta: 10 junio 2018].

»McAllister, David; Nancy McAllister y Steve Vivian. 2002. The impact of digital books upon print publishing. En IEEE 2002 International Symposium on Technology and Society (ISTAS'O2). Social Implications of Information and Communication Technology. Proceedings (Cat. No.02CH37293). p. 150-154. https://doi. org/10.1109/ISTAS.2002.1013810

》McGrath, Rita Gunther. 2010. Business Models: A Discovery Driven Approach. En Long Range Planning. Vol. 43, 247-261. https://doi.org/10.1016/j. Irp.2009.07.005

" Morris, Langdon. 2009. Business Model Innovation: The Strategy of Business Breakthroughs. En International Journal of Innovation Science. Vol. 1, no.4, 191204. https://doi.org/10.1260/1757-2223.1.4.191

" Øiestad, Sara y Markus Bugge. 2014. Digitisation of publishing: exploration based on existing business models. En Technological Forecasting and Social Change. Vol. 83, 54-65. https://doi.org/10.1016/j.techfore.2013.01.010

»Peng, Ying. 2016. Mobile and digitally-mediated publishing strategies in China: an overview of evolving business models. En Publishing Research Quarterly. Vol. 32, no. 3, 247-26o.

"Porter, Michael. 2001. Strategy and the Internet. En Harvard Business Review. Vol. 79, no. 3, 62-78.

"Protogerou, Aimilia; Alexandra Kontolaimou y Yannis Caloghirou. 2017. Innovation in the European creative industries: a firm-level empirical approach. En Industry and Innovation. Vol. 24, no. 6, 587-612. https://doi.or g/10.1080/13662716.2016.1263551

»Rayna, Thierry y Ludmila Striukova. 2016. From rapid prototyping to home fabrication: How $3 \mathrm{D}$ printing is changing business model innovation. En Technological Forecasting and Social Change. Vol. 102, 214-224. https://doi.org/10.1016/j. techfore.2015.07.023

»Rayport, Jeffrey y John Sviokla. 1995. Exploiting the virtual value chain. En Harvard Business Review. Vol. 73, no. 6, 35-51.

»Sabatier, Lou Ann y Edward Fitzelle. 2011. Managing the progressive publishing company during market and technology transitions. En Publishing Research Quarterly. Vol. 27, 220-229.

» Sánchez Tarragó, Nancy y Yuniar Díaz Álvarez. 2005. El sector editorial contemporáneo y las competencias profesionales. En Acimed. Vol. 13, no. 5, 1-5.

»Senftleben, Martin; Maximilian Kerk, Miriam Buiten y Klaus Heine. 2017. From books to content platforms-new business models in the Dutch publishing sector. (January 16, 2017). Amsterdam: SSRN. http://dx.doi.org/10.2139/ssrn.2904116

»Shatzkin, Mike. 2008. Digital publishing in the US: driving the industry to vertical niches?. En Logos. Vol. 19, no. 2, 56-60. http://dx.doi.org/10.2959/logo.2008.19.2.56 
»Tian, Xuemei y Bill Martin. 2010. Digital technologies for book publishing. En Publishing Research Quarterly. Vol. 26, no. 3, 151-167.

»Tian, Xuemei; Bill Martin y Hepu Deng. 2008. The impact of digitization on business models for publishing. En Journal of Systems and Information Technology. Vol. 10, no. 3, 232-25o. https://doi.org/10.1108/13287260810916934

» Tushman, Michael y Philip Anderson. 1986. Technological discontinuities and organizational environments. En Administrative Science Wuarterly. Vol. 31, no. 3 , 439-465. https://doi.org/10.2307/2392832

»Wischenbart, Rüdiger. 2016. The Global eBook Report. Vienna: Rüdiger Wischenbart Content and Consulting.

"Zott, Christoph y Raphael Amit. 2007. Business model design and the performance of intrepreneurial firms. En Organization Science. Vol. 18, no. 2, 181-199. https://doi.org/10.1287/orsc.1060.0232

\section{Fuentes consultadas}

»Fedecali. 2010. Comercio exterior del libro 2009. Madrid: Federación Española de Cámaras del Libro.

»Fedecali. 2011. Comercio exterior del libro 2010. Madrid: Federación Española de Cámaras del Libro.

»Fedecali. 2012. Comercio exterior del libro 2011. Madrid: Federación Española de Cámaras del Libro.

»Fedecali. 2013. Comercio exterior del libro 2012. Madrid: Federación Española de Cámaras del Libro.

» Fedecali. 2014. Comercio exterior del libro 2013. Madrid: Federación Española de Cámaras del Libro.

» Fedecali. 2015. Comercio exterior del libro 2014. Madrid: Federación Española de Cámaras del Libro.

»Fedecali. 2016. Comercio exterior del libro 2015. Madrid: Federación Española de Cámaras del Libro.

»Fedecali. 2017. Comercio exterior del libro 2016. Madrid: Federación Española de Cámaras del Libro.

» FGEE. 1998. Comercio interior del libro 1997. Madrid: Federación de Gremios de Editores de España (FGEE).

» FGEE. 1999. Comercio interior del libro 1998. Madrid: Federación de Gremios de Editores de España (FGEE).

» FGEE. 2000. Comercio interior del libro 1999. Madrid: Federación de Gremios de Editores de España (FGEE).

» FGEE. 2001. Comercio interior del libro 2000. Madrid: Federación de Gremios de Editores de España (FGEE). 
"FGEE. 2002. Comercio interior del libro 2001. Madrid: Federación de Gremios de Editores de España (FGEE).

》FGEE. 2003. Comercio interior del libro 2002. Madrid: Federación de Gremios de Editores de España (FGEE).

» FGEE. 2004. Comercio interior del libro 2003. Madrid: Federación de Gremios de Editores de España (FGEE).

"FGEE. 2005. Comercio interior del libro 2004. Madrid: Federación de Gremios de Editores de España (FGEE).

》FGEE. 2006. Comercio interior del libro 2005. Madrid: Federación de Gremios de Editores de España (FGEE).

" FGEE. 2007. Comercio interior del libro 2006. Madrid: Federación de Gremios de Editores de España (FGEE).

» FGEE. 2008. Comercio interior del libro 2007. Madrid: Federación de Gremios de Editores de España (FGEE).

》FGEE. 2009. Comercio interior del libro 2008. Madrid: Federación de Gremios de Editores de España (FGEE).

» FGEE. 2010. Comercio interior del libro 2009. Madrid: Federación de Gremios de Editores de España (FGEE).

» FGEE. 2011. Comercio interior del libro 2010. Madrid: Federación de Gremios de Editores de España (FGEE).

» FGEE. 2012. Comercio interior del libro 2011. Madrid: Federación de Gremios de Editores de España (FGEE).

» FGEE. 2014. Comercio interior del libro 2013. Madrid: Federación de Gremios de Editores de España (FGEE).

» FGEE. 2015. Comercio interior del libro 2014. Madrid: Federación de Gremios de Editores de España (FGEE).

» FGEE. 2016. Comercio interior del libro 2015. Madrid: Federación de Gremios de Editores de España (FGEE).

» FGEE. 2017. Comercio interior del libro 2016. Madrid: Federación de Gremio de Editores de España (FGEE).

»Ministerio de Educación, Cultura y Deporte. 1997. Panorámica de la edición española de libros 1996. Análisis sectorial del libro. Madrid: Secretaría General Técnica.

" Ministerio de Educación, Cultura y Deporte. 1998. Panorámica de la edición española de libros 1997. Análisis sectorial del libro. Madrid: Secretaría General Técnica.

" Ministerio de Educación, Cultura y Deporte. 1999. Panorámica de la edición española de libros 1998. Análisis sectorial del libro. Madrid: Secretaría General Técnica.

» Ministerio de Educación, Cultura y Deporte. 2000. Panorámica de la edición española de libros 1999. Análisis sectorial del libro. Madrid: Secretaría General Técnica.

» Ministerio de Educación, Cultura y Deporte. 2001. Panorámica de la edición española de libros 2000. Análisis sectorial del libro. Madrid: Secretaria General Técnica. 
" Ministerio de Educación, Cultura y Deporte. 2002. Panorámica de la edición española de libros 2001. Análisis sectorial del libro. Madrid: Secretaría General Técnica.

» Ministerio de Educación, Cultura y Deporte. 2003. Panorámica de la edición española de libros 2002. Análisis sectorial del libro. Madrid: Secretaría General Técnica.

» Ministerio de Educación, Cultura y Deporte. 2004. Panorámica de la edición española de libros 2003. Análisis sectorial del libro. Madrid: Secretaría General Técnica.

» Ministerio de Educación, Cultura y Deporte. 2005. Panorámica de la edición española de libros 2004. Análisis sectorial del libro. Madrid: Secretaría General Técnica.

» Ministerio de Educación, Cultura y Deporte. 2006. Panorámica de la edición española de libros 2005. Análisis sectorial del libro. Madrid: Secretaría General Técnica.

" Ministerio de Educación, Cultura y Deporte. 2007. Panorámica de la edición española de libros 2006. Análisis sectorial del libro. Madrid: Secretaría general Técnica.

» Ministerio de Educación, Cultura y Deporte. 2008. Panorámica de la edición española de libros 2007. Análisis sectorial del libro. Madrid: Secretaría General Técnica.

»Ministerio de Educación, Cultura y Deporte. 2009. Panorámica de la edición española de libros 2008. Análisis sectorial del libro. Madrid: Secretaría General Técnica.

» Ministerio de Educación, Cultura y Deporte. 2010. Panorámica de la edición española de libros 2009. Análisis sectorial del libro. Madrid: Secretaría General Técnica.

» Ministerio de Educación, Cultura y Deporte. 2011. Panorámica de la edición española de libros 2010. Análisis sectorial del libro. Madrid: Secretaría General Técnica.

» Ministerio de Educación, Cultura y Deporte. 2012. Panorámica de la edición española de libros 2011. Análisis sectorial del libro. Madrid: Secretaría General Técnica.

»Observatorio de la Lectura y el Libro. 2013. Panorámica de la edición española de libros 2012. Análisis sectorial del libro. Madrid: Secretaría General Técnica.

»Observatorio de la Lectura y el Libro. 2014. Panorámica de la edición española de libros 2013. Análisis sectorial del libro. Subdirección General de Documentación y Publicaciones. Madrid: Secretaría General Técnica.

"Observatorio del la Lectura y el Libro. 2015. Panorámica de la edición española de libros 2014. Análisis sectorial del libro. Madrid: Secretaría General Técnica.

"Observatorio de la Lectura y el Libro. 2016. Panorámica de la edición española de libros 2015. Análisis sectorial del libro. Madrid: Secretaría General Técnica. 